\title{
Eigenverantwortung im Gesundheitsrecht ${ }^{1}$
}

\section{STEFAN HUSTER}

Stefan Huster ist Professor für Öffentliches Recht und Sozialrecht an der Juristischen Fakultät der RuhrUniversität Bochum und Direktor des Instituts für Gesundheits- und Sozialrecht

\author{
Sowohl im System der medizinischen Versorgung \\ als auch im Rahmen der Präventions- und \\ Gesundheitsförderungspolitik stellt sich die \\ Frage, welche Bedeutung dem Grundsatz der \\ Eigenverantwortung zukommt. Der Beitrag \\ vergleicht die Diskussionen in beiden Bereichen und \\ stellt abschließend ihren Zusammenhang dar.
}

\section{Gesundheitsversorgung und Public Health}

\subsection{Gesundheit als besonderes Gut}

Gesundheit ist ein besonderes Gut. Gesundheitliche Beeinträchtigungen haben eine existentielle Dimension, die andere soziale Defizite nicht in der gleichen Weise besitzen: So schlimm es sein mag, etwa keine Arbeit zu haben oder sein Leben mit sehr begrenztem Einkommen fristen zu müssen, so unvergleichbar intensiver ist die Erfahrung einer schweren, vielleicht sogar lebensbedrohenden Krankheit und der damit verbundenen Schmerzen, Beeinträchtigungen und psychischen Belastungen. Gesundheit ist daneben ein konditionales oder - im kantischen Sinne des Wortes - transzendentales Gut, weil es die Voraussetzung für viele andere Lebensvollzüge darstellt. Der Volksmund weiß: »Gesundheit ist nicht alles, aber ohne Gesundheit ist alles nichts." Gegen eine Einschränkung der Lebensführung durch gesundheitliche Probleme angehen zu können, soweit dies medizinisch möglich ist, ist gerade in einer Wettbewerbs- und Leistungsgesellschaft von zentraler Bedeutung, weil dadurch eine Chancen- oder Startgleichheit hergestellt wird, die die differenzierten Ergebnisse dieses Wettbewerbsprozesses erst legitimiert ( $\mathrm{Da}$ niels 1985). Dies dürfte auch erklären, warum - ähnlich wie im Bereich der Bildung - gegen soziale Differenzierun- gen der Gesundheit massive politische Vorbehalte bestehen.

\subsection{Das System der medizinischen Versorgung}

Es gibt daher gute Gründe, den Sozialstaat auch und sogar vorrangig auf die Erhaltung der Gesundheit seiner Bürger zu verpflichten. In diesem $\mathrm{Zu}$ sammenhang richtet sich zumindest in Deutschland die Aufmerksamkeit fast ausschließlich auf die Gewährleistung der medizinischen Versorgung. Auch die Rechtsprechung des Bundesverfassungsgerichts weist der Gewährleistung der medizinischen Versorgung einen außerordentlich hohen Stellenwert zu. Lange Zeit hatte sich das Verfassungsgericht eher zurückhaltend in dem Sinne geäußert, dass sich aus den Grundrechten und dem Sozialstaatsprinzip kein Anspruch auf die Bereithaltung spezieller Gesundheitsleistungen ergibt; die objektivrechtliche Pflicht des Staates, sich schützend und fördernd vor das Rechtsgut des Lebens und der körperlichen Unversehrtheit (Art. 2 Abs. 2 S. 1 Grundgesetz - GG) zu stellen, sei unter Berücksichtigung der Gestaltungsfreiheit der zuständigen staatlichen Stellen lediglich darauf gerichtet, dass die öffentliche

\footnotetext{
1 Der Aufsatz ist bereits in der Zeitschrift Ethik in der Medizin 22 (2010), S. 289-299, erschienen.
} 
Gewalt Vorkehrungen zum Schutz des Grundrechts trifft, die nicht völlig ungeeignet oder unzulänglich sind. Neuere Entscheidungen haben die verfassungsrechtlichen Anforderungen aber deutlich erhöht, wenn aus der allgemeinen Handlungsfreiheit (Art. 2 Abs. 1 GG) i.V.m. dem Sozialstaatsprinzip sowie aus Art. 2 Abs. 2 GG ein Leistungsanspruch des Versicherten in der Gesetzlichen Krankenversicherung (GKV) auch auf noch nicht anerkannte Behandlungsmethoden abgeleitet wird, wenn für dessen lebensbedrohliche oder regelmäßig tödliche Erkrankung eine allgemein anerkannte, medizinischem Standard entsprechende Behandlung nicht zur Verfügung steht und eine nicht ganz entfernt liegende Aussicht auf Heilung oder auf eine spürbare positive Einwirkung auf den Krankheitsverlauf durch die von ihm gewählte und ärztlich angewandte Behandlungsmethode besteht (BVerfG 2005). Eine weitere Verschärfung ergibt sich daraus, dass es das Bundesverfassungsgericht ausdrücklich für möglich erachtet hat, dass sich dieser Anspruch auch auf Methoden beziehen kann, über deren Zulassung zur Versorgung in der GKV der Gemeinsame Bundesausschuss als das zentrale Steuerungsorgan bereits negativ entschieden hat (BVerfG 2007); diese Auffassung hat der Gesetzgeber nun ausdrücklich in das SGB V übernommen. Die gesetzlich zur Entscheidung berufenen Organe können also tun, was sie wollen - jedenfalls bei einer lebensbedrohlichen Krankheit scheint sich der "Höchstwert Leben " (BVerfG 2005) in Form grundrechtsunmittelbarer Ansprüche Bahn zu brechen.

\subsection{Die Bedeutung anderer Faktoren für Gesundheit und Krankheit}

Diese Fokussierung der Aufmerksamkeit auf das Versorgungssystem und die parallele Verschärfung der verfassungsrechtlichen Vorgaben wird man mit Blick auf die Opportunitätskosten (vgl. dazu Huster; Kliemt 2009) nicht uneingeschränkt begrüßen, wenn man sich vergegenwärtigt, dass das System der medizinischen Versorgung weder für die Gesundheit der Bevölkerung - traditionell formuliert: die Volksgesundheit - noch für die soziale Verteilung von Gesundheit - die »Gesundheitsgerechtigkeit« - von zentraler Bedeutung ist.
Tatsächlich weisen zahlreiche Untersuchungen darauf hin, dass für den Gesundheitszustand einer Population maßgeblich andere, nämlich - im weitesten Sinne - soziale Faktoren verantwortlich sind, nämlich - neben dem Zugang zur Gesundheitsversorgung und der genetischen Konstitution - die Verhältnisse am Arbeitsplatz und im Wohnumfeld, das individuelle Gesundheitsverhalten sowie originär sozialstrukturelle Faktoren (vgl. dazu nur Mielck 2000 und Richter; Hurrelmann 2006).

\section{Gesundheitsrelevante Faktoren sind zu einem erheblichen Teil schichtenspezifisch ausgeprägt.}

Diese Faktoren dürften auch maßgeblich den sozialen Gesundheitsgradienten erklären. Es lässt sich nämlich nachweisen, dass diese gesundheitsrelevanten Faktoren zu einem erheblichen Teil schichtenspezifisch ausgeprägt sind: Angehörige der unteren sozialen Schichten sind - wie die einschlägigen Studien zeigen - häufiger einem gesundheitlich belastenden Arbeitsumfeld ausgesetzt und leiden auch in ihrem Wohnumfeld häufiger unter Lärm und Luftverschmutzung; sie ernähren sich ungesünder, neigen stärker zu Nikotinund übermäßigem Alkoholkonsum und vernachlässigen die körperliche Bewegung; schließlich leiden sie sowohl in ihrem Privat- und Arbeitsleben als auch in der (politischen) Öffentlichkeit an geringen Kontroll- und Selbstbestimmungsmöglichkeiten. Jedenfalls können die sozialen Gesundheitsungleichheiten zumindest in Deutschland gewiss nicht ausschließlich auf den Unterschied von gesetzlicher und privater Krankenversicherung oder auf soziale Zugangsbarrieren zur gesundheitlichen Versorgung zurückgeführt werden.

\subsection{Das Aufmerksamkeitsdefizit der Public Health-Politik}

Angesichts des Umstandes, dass die sozialen Gesundheitsfaktoren sowohl für die Volksgesundheit als auch für die Gesundheitsverteilung von maßgeblicher Bedeutung sind, stellen die Protagonis- ten einer auf diese Faktoren bezogenen Public Health-Politik immer wieder geradezu verzweifelt die Frage, warum dieses Politikfeld im Unterschied zur Gesundheitsversorgung eine erheblich geringere Aufmerksamkeit genießt. Dieses Aufmerksamkeitsdefizit kommt auch im Recht zum Ausdruck. Gewiss gibt es ganze Rechtsgebiete, die auch oder sogar vorrangig dem Gesundheitsschutz dienen; erwähnt seien nur das Umweltrecht und das Recht der Sicherheit am Arbeitsplatz. Schon mit Blick auf die Bekämpfung gesundheitlicher Ungleichheiten fällt die Bilanz aber dürftig aus: Soweit ersichtlich findet sich allein in \$ 20 Abs. 1 S. 2 Fünftes Buch Sozialgesetzbuch (SGB V) der Hinweis, dass Leistungen der Primärprävention nicht nur den allgemeinen Gesundheitszustand verbessern, sondern insbesondere auch die sozial bedingte Ungleichheit von Gesundheitschancen vermindern sollen.

Verantwortlich für dieses Aufmerksamkeitsdefizit dürfte eine ganze Reihe von Aspekten sein, zu denen zunächst die »politische Ökonomie« der Gesundheitspolitik gehört. Wie jede Politik, die ein öffentliches Gut fördert, das im allgemeinen Interesse liegt, hat auch die Public Health-Politik ein Problem der politischen Wahrnehmung und Durchsetzung (Burris 1997). Es existiert hier - anders als im "Medizinsystem « - keine Lobby, für die die Ausweitung der Ressourcen oberste Priorität besitzt. Hinzu kommt, dass die technische Faszination, die von der modernen Medizin ausgeht, der Public Health-Politik fehlt. Und: Prävention kostet zunächst einmal Geld; ob damit mittel- und langfristig Kosten im Gesundheitswesen eingespart werden können, ist durchaus umstritten (Beske 2002). Aufgrund des Populationsbezugs von Public Health-Maßnahmen ist schließlich auch die Motivation der einzelnen Bürger, sich für diese Maßnahmen politisch einzusetzen, nicht sehr stark ausgeprägt. Während Leistungen der Gesundheitsversorgung einen klaren Individualbezug haben und deshalb die Aufmerksamkeit der Bürger auf sich ziehen, schlägt im Bereich der Public Health das sog. Präventionsparadox durch: Auf einer statistischen Ebene haben schon geringe Veränderungen des Verhaltens oder der Umwelt deutliche Auswirkungen auf die Volksgesundheit, auf der individuellen Ebene sind diese 
Effekte dagegen sehr gering und bringen dem einzelnen nur einen marginalen Vorteil (Rose 1985). Es ist daher nicht überraschend, dass sie in der demokratischen Öffentlichkeit kein allzu großes Interesse finden.

Diese Einstellungen werden durch die Struktur eines Rechtssystems verstärkt, das der individuellen Freiheit und individuellen Rechten einen normativen Vorrang einräumt. Die Gefährdungen konkreter Lebens- und Gesundheitsinteressen, die im Rahmen der medizinischen Versorgung angegangen werden, lassen sich über individuelle Rechte sehr viel leichter thematisieren als die statistischen Wirkungen einer Public HeathPolitik. Das Rechtssystem verschärft dadurch die asymmetrische Einstellung gegenüber gefährdeten konkreten und statistischen Leben, die in unseren moralischen Intuitionen bereits eine große Rolle spielt (Huster 2008). Ähnliches gilt für die Verteilungswirkungen: Während die sozial differenzierenden Auswirkungen vieler gesundheitspolitischer Steuerungsinstrumente im Versorgungssystem - etwa der Praxisgebühr oder der Erhöhung von Zuzahlungen - auf der Hand liegen und entsprechend heftig diskutiert werden, bleiben die Effekte der allgemeinen Gesellschaftspolitik - so gesundheitsrelevant sie auch sein mögen - häufig diffus. Es ist daher vielleicht kein Zufall, dass Public Health in Institutionen wie der EU und der WHO, denen man ein technokratisches Politikverständnis nachsagt und in denen die gesundheitspolitische Diskussion schon mangels entsprechender Kompetenzen nicht durch die Verteilungskämpfe in den Systemen der medizinischen Versorgung bestimmt wird, eine größere Rolle spielt.

\subsection{Der Einwand des Paternalismus}

Die größten juristischen Vorbehalte gegen eine Public Health-Politik dürften sich allerdings aus einem Aspekt ergeben, der im Folgenden näher diskutiert werden soll: Wenn und soweit diese Politik auf die lebensstilbezogenen Gesundheitsfaktoren bezogen ist, scheint sie unweigerlich einen freiheitsfeindlichen und paternalistischen $\mathrm{Zug} \mathrm{zu}$ erhalten. Sie könnte daher in eine präventionsstaatliche Gesundheitsdiktatur des »healthism« umschlagen, wenn sie auf die gesundheitsrelevanten Lebens- gewohnheiten der Bürger Einfluss zu nehmen sucht. Dabei wird die Frage, welche Rolle die Eigenverantwortung der Bürger für ihre Gesundheit spielen sollte, inner- und außerhalb des Versorgungssystems durchaus unterschiedlich diskutiert.

\section{Eigenverantwortung in der Gesundheitsversorgung}

\subsection{Eigenverantwortung im Krankenversicherungsrecht}

Der Grundsatz der Eigenverantwortung hat im Recht der öffentlichen medizinischen Versorgung lange Zeit ein Schattendasein gefristet. $\int 1$ SGB V betont zwar programmatisch neben dem Solidarprinzip auch die Eigenverantwortung der Versicherten für ihre Gesundheit, für die Leistungsansprüche der Versicherten hatte dies bislang aber kaum eine rechtspraktische Bedeutung. Die Krankenversicherung orientiert sich nämlich nach allgemeiner Auffassung am Finalprinzip, auf die Ursache der Krankheit kommt es für den Leistungsanspruch demnach nicht an (Muckel 2009). Dies stimmt damit zusammen, dass $\mathbb{S} 52$ Abs. 1 SGB V den Leistungsanspruch bei Selbstverschulden nur für den Fall begrenzt, dass sich der Versicherte die Krankheit vorsätzlich zugezogen hat. Dies ist aber typischerweise selbst bei einem extrem gesundheitsgefährdenden Verhalten nicht der Fall, da der Betroffene hoffen wird, dass gerade in seinem Fall die Gesundheitsbeschädigung nicht eintritt, so dass von einer vorsätzlichen Zuziehung keine Rede sein kann. Die Norm hat daher kaum einen Anwendungsbereich.

Einen Vorstoß zur stärkeren Berücksichtigung der Eigenverantwortung hat der Gesetzgeber allerdings 2007 mit dem GKV-Wettbewerbsstärkungsgesetz unternommen: Nach dem neu eingefügten Abs. 2 des $\mathbb{S} 52$ SGB V waren Versicherte an den Behandlungskosten zu beteiligen, wenn sie sich »eine Krankheit durch eine medizinisch nicht indizierte Maßnahme wie zum Beispiel eine ästhetische Operation, eine Tätowierung oder ein Piercing zugezogen« haben. Streng genommen waren damit aufgrund der nur exemplarischen Aufzählung (»wie zum Beispiel «) auch gesundheitliche Folgeprobleme z. B. des verbreiteten und sozial unauffälligen Durchstechens der Ohrläppchen, um Ohrschmuck tragen zu können, kostenbeteiligungspflichtig. Das war dem Gesetzgeber aber wohl zuviel des Guten, und so wurde durch das Pflege-Weiterentwicklungsgesetz ab dem 1.7.2008 »klargestellt" (so die Gesetzesbegründung), dass die Beteiligungspflicht auf die ausdrücklich genannten drei Maßnahmen beschränkt ist, indem die Formulierung "Maßnahme wie zum Beispiel eine « in $\$ 52$ Abs. 2 SGB V gestrichen wurde. Nun tritt allerdings zum einen ein erhebliches Gleichheitsproblem auf: Warum sind die Folgeprobleme von ästhetischen Operationen, Tattoos und Piercings kostenbeteiligungspflichtig, nicht aber die gesundheitlichen Konsequenzen vergleichbarer Maßnahmen wie des Branding, Cutting und der besagten Ohrringe (Höfling 2009 und Wienke 2009)? Zum anderen fragt es sich, wie die Krankenkassen überhaupt davon erfahren, dass eine Krankheit auf
Es ist daher vielleicht kein Zufall, dass Public Health in Institutionen wie der EU und der WHO eine größere Rolle spielt.

einen der in $\mathbb{5} 52$ Abs. 2 SGB V genannten Eingriffe zurückgeht. Dazu hat der Gesetzgeber in $\mathbb{} 294 a$ Abs. 2 SGB V inzwischen eine entsprechende Mitteilungspflicht der an der vertragsärztlichen Versorgung teilnehmenden Ärzte und Krankenhäuser eingeführt; dies ist mit Blick auf das Vertrauensverhältnis zwischen Arzt und Patient ersichtlich eine problematische Regelung.

Ebenfalls durch das GKV-Wettbewerbsstärkungsgesetz hat der Gesetzgeber die Bedeutung der präventionsbezogenen Eigenverantwortung gestärkt, indem er die Absenkung der Zuzahlungsbelastungsgrenze für chronisch Kranke von 2 auf $1 \%$ des Jahresbruttoverdienstes an die Voraussetzung knüpft, dass die Betroffenen regelmäßig an bestimmten Untersuchungen teilnehmen ( $\int 62$ Abs. 1 S. 3 SGB V). Bereits seit längerer Zeit ist die Höhe des Zuschusses der Krankenkasse für Zahnersatz davon abhängig, ob sich der 
Versicherte regelmäßig hat zahnärztlich untersuchen lassen ( $\$ 55$ Abs. 1 SGB V).

\subsection{Vorbehalte gegen das Kriterium}

Wer sich freiwillig dafür entschieden hat, ein bestimmtes Risiko einzugehen, wird sich grundsätzlich nicht darüber beschweren können, dass er die Folgekosten trägt oder zumindest an ihnen beteiligt wird, wenn sich dieses Risiko realisiert. Anders als im Fall des »brute luck«, wenn ein unverschuldetes Risiko eintritt, kann es die Eigenverantwortung des Betroffenen in dieser Konstellation des »option luck « rechtfertigen, dass die Solidargemeinschaft ihm die Bewältigung der Folgeprobleme überlässt (zur Begrifflichkeit vgl. Dworkin 1981). Dies gilt im Grundsatz auch für die Gesundheitsversorgung. Dass bisher im Krankenversicherungsrecht darauf verzichtet worden ist, dem Gedanken der Eigenverantwortung eine zentrale Bedeutung zuzusprechen, zeigt allerdings, dass in diesem Bereich besondere Schwierigkeiten auftreten können. Auch die Diskussion über Priorisierungs- und Rationierungskriterien hat sich für das Kriterium der Eigenverantwortung oder des Selbstverschuldens nicht erwärmen können (zur Diskussion vgl. Alber et al 2009 und Buyx 2005).

Dabei dürfte das größte Problem nicht darin bestehen, dass es im Einzelfall schwierig oder gar unmöglich sein mag, eine Erkrankung auf ein bestimmtes Verhalten kausal zurückzuführen. Dies ist in der Sache zwar richtig, hindert das Recht aber nicht von vornherein an Verantwortungszuschreibungen; selbst im Strafrecht kennen wir abstrakte Gefährdungsdelikte, für deren Verwirklichung ein konkreter Schaden eben nicht eingetreten sein muss. Es ist daher nicht klar, warum nicht auch im Krankenversicherungsrecht ein risikoerhöhendes Verhalten zwar nicht zu einem Leistungsausschluss, wohl aber zu finanziellen Auswirkungen sollte führen dürfen. Die Differenzierung der Zuzahlungsbelastungsgrenze in $\mathbb{S} 62$ Abs. 1 SGB V beruht ersichtlich auf diesem Gedanken, denn auch hier wird man im Einzelfall nicht immer eindeutig sagen können, dass der Krankheitsverlauf günstiger gewesen wäre, wenn der Versicherte an den Gesundheitsuntersuchungen teilgenommen hätte. Allerdings - dies zeigt die Diskussion des $\mathbb{} 52$ Abs. 2 SGB V - müssen die risiko- und kostenerhöhenden Tatbestände willkürfrei ausgewählt werden. Zudem wird man - wenn es wirklich um den Ausgleich zwischen individueller und kollektiver Kostenverantwortung und nicht um einen versteckten gesundheitspolitischen Paternalismus geht - vorurteilsfrei errechnen müssen, welche Kosten, aber auch Ersparnisse, eine selbstverschuldete Krankheit für das Gemeinwesen mit sich bringt.

Die eigentlichen Probleme dürften aber an anderer Stelle liegen. Das Beispiel des $\mathbb{5} 52$ Abs. 2 SGB V zeigt, dass die Berücksichtigung des Gesundheitsverhaltens im Versorgungssystem zum einen mit intrikaten Ermittlungen in der

\section{Die Berücksichtigung des Gesundheitsverhaltens kann mit intrikaten Ermittlungen in der persönlichen Lebenssphäre verbunden sein.}

persönlichen Lebenssphäre verbunden sein kann. Zum anderen kann man darüber streiten, wie frei jeweils die Entscheidung für ein gesundheitsgefährdendes Verhalten ist. Während punktuelle gesundheitsriskante Entscheidungen etwa zur Vornahme eines Piercings oder zur Ausübung einer besonders unfallträchtigen Sportart - in der Regel ohne weiteres mitsamt ihren Konsequenzen der Selbstverantwortung zugeschrieben werden können, haben die gerade für viele chronische Krankheiten relevanten Lebensstile wie das Ernährungsund Bewegungsverhalten regelmäßig eine sehr viel längere und kompliziertere Genese. An dieser Stelle wird es nun relevant, wie sich Politik und Recht zu diesen Umständen verhalten.

\section{Eigenverantwortung und Public Health}

Während Ansätze, der Eigenverantwortung im Rahmen der Gesundheitsversorgung eine stärkere Rolle zuzuschreiben, vielfach als »Entsolidarisierung " kritisiert werden (Wienke 2009), weil sie die individuelle im Verhältnis zur kollektiven Verantwortung zu sehr betonten, nimmt die Diskussion der Public Health-Politik häufig einen anderen Verlauf: Soweit sich diese Politik auf die Verhaltensprävention konzentriert, ist sie dem Einwand ausgesetzt, die Autonomie und Eigenverantwortung der Bürger nicht ernst genug zu nehmen.

\subsection{Gesundheitsungleichheiten und Gerechtigkeit}

Dabei ist dieser Einwand zum einen für die Frage von Bedeutung, ob Staat und Politik zum Tätigwerden verpflichtet sind. Dies wird insbesondere mit Blick auf die als anstößig empfundenen sozialen Gesundheitsungleichheiten häufig angenommen. Soweit sich gesundheitliche Auswirkungen und Unterschiede aber aus der individuellen Lebensführung ergeben, scheinen sie keine Handlungspflicht des Sozialstaats zu begründen: Wenn einzelne Bürger oder auch ganze Bevölkerungsschichten ihre Gesundheit durch ihr frei gewähltes Verhalten ruinieren, stellt das weder ein normatives Problem dar, noch begründet es politisch-kollektive Verantwortlichkeiten. In einer freiheitlichen Gesellschaft ist es grundsätzlich alles andere als sachfremd, den Bürgern die Verantwortung für die (finanziellen) Folgen ihrer freien Entscheidungen zuzuschreiben. Selbst egalitaristisch gesonnene Theorien der Gerechtigkeit vertreten die Ansicht, eine gerechte Güterverteilung sei zwar »endowment-insensitive ", aber "ambition-sensitive «: Sie müsse die unverdienten Vor- und Nachteile ausgleichen, die sich aus den unterschiedlichen Startbedingungen und individuell nicht beeinflussbaren Umweltbedingungen ergeben, nicht aber die differenzierten Folgen der autonomen Entscheidungen (Dworkin 1981). Rechtliche Freiheit führt eben zu tatsächlicher Ungleichheit.

Nun trifft dieser Einwand nicht die gesamte Public Health-Politik, weil sich die sozialen Gesundheitsunterschiede nicht ausschließlich und vermutlich nicht einmal vorrangig als Auswirkungen des Gesundheitsverhaltens erklären lassen. Soweit diese Politik aber auf dieses Verhalten bezogen ist, scheint der Einwand, dass hier keine korrekturbedürftige Ungerechtigkeit vorliegt, zumindest auf den ersten Blick richtig zu sein. 


\subsection{Rechtfertigung der Beeinflussung des Gesundheitsverhaltens}

Schlimmer noch: Unter der genannten Prämisse wäre staatliches Handeln nicht nur nicht geboten, sondern sogar erheblichen normativen Einwänden ausgesetzt. Es fragt sich nämlich sehr ernsthaft, welche Legitimation die öffentliche Gewalt besitzt, um auf die gesundheitsrelevante Lebensführung der Bürger einzuwirken.

Am nächsten liegt das erste Argument, dass das Gemeinwesen ein Interesse an einer gesundheitsbewussten Lebensführung hat, weil dadurch Kosten reduziert werden (vgl. etwa Händeler 2008). Dies ist ein moralisch zunächst unverdächtiger Ansatz, weil er auf dem bekannten Grundsatz beruht, dass externe Kosten zu internalisieren sind (Eichenhofer 2003). Soweit damit die Kosten der Sozialsysteme und insbesondere der medizinischen Versorgung gemeint sind, ist aber zum einen nicht sehr klar, ob und unter welchen Umständen dies zutreffend ist. Bekanntlich könnte es sein, dass etwa das Rauchen für die sozialen Systeme letztlich kostenneutral oder sogar kostensparend ist. Zum anderen scheint die Beeinflussung der Lebensstile der falsche Ansatzpunkt zu sein; konsequent wäre es, die Betroffenen unmittelbar an den Behandlungskosten ihrer Krankheiten zu beteiligen. Soweit man in die zu berücksichtigenden Kosten darüber hinaus auch den Arbeitsausfall und sonstige gesellschaftliche Folgekosten einbezieht, wird man berücksichtigen müssen, dass dieses Vorgehen einer freiheitlichen Gesellschaftsordnung eher fremd ist: Denn auch unabhängig vom Gesundheitsverhalten haben die Bürger hier keine Pflicht, sich zu möglichst produktiven Mitgliedern des Gemeinwesens zu entwickeln. Wer etwa seine Talente verkümmern lässt, anstatt sie auszubilden und für das Gemeinwesen einzusetzen, wird zwar typischerweise ein geringeres Markteinkommen erzielen, er wird aber nicht zusätzlich mit einer Strafabgabe belegt.

Ein zweites Argument, das grundsätzlich unverdächtig ist, aber ebenfalls über eine sehr begrenzte Reichweite verfügt, stellt darauf $a b$, dass ein riskantes Verhalten nicht nur die Gesundheit des Betroffenen, sondern auch das Wohlergehen Dritter gefährden kann.
Auf diesem Wege sind insbesondere die gesetzlichen Beschränkungen des Tabakkonsums in der Öffentlichkeit gerechtfertigt worden. Die dazu ergangene Rechtsprechung zeigt allerdings, dass der Schutz der Nichtraucher ausnahmslose Rauchverbote schwerlich trägt: Sind Nichtraucher gar nicht, nur marginal oder mit ihrem Einverständnis betroffen, gerät dieses Argument schnell an seine Grenzen. Soweit das Bundesverfassungsgericht trotzdem strikte Rauchverbote für zulässig hielt (BVerfG 2008), musste es sich auch bereits heftige Kritik gefallen lassen (vgl. nur Gröschner 2008). Selbst innerhalb des Gerichts war umstritten, ob damit nicht »ein Weg edukatorischer Bevormundung vorgezeichnet (wird), der sich auf weitere Bereiche ausdehnen könnte und dann erstickend wirkt « (BVerfG 2008). Zudem gibt es zahlreiche gesundheitsgefährdende Verhaltensweisen, die auf Dritte gar keine Auswirkungen haben: Wer sich falsch ernährt oder zu wenig bewegt, schädigt in keinem relevanten Sinne das Wohlergehen Dritter.

Die einschlägigen politischen Maßnahmen tragend, aber in seinem normativen Status heikel ist ein drittes Argument, nach dem es darum geht, die Gesundheit der Bevölkerung insgesamt zu schützen und zu verbessern. Dieser Hinweis auf die "Volksgesundheit « hat in Deutschland aus historischen Gründen schon begrifflich einen besonders schweren Stand (vgl. nur Frenzel 2007). Aber auch unabhängig davon stellt sich tatsächlich die Frage, ob es ein derartiges Rechtsgut in einer freiheitlichen Ordnung geben kann. Soweit es um die Folgekosten von Krankheiten oder eine Drittschädigung - etwa bei übertragbaren Krankheiten - geht, lässt sich dieser Ansatz auf die beiden bereits erwähnten Argumente reduzieren. Darüber hinaus scheint Gesundheit aber tatsächlich ein privates Gut zu sein, so dass sich die »Volksgesundheit« nur als summative Bezeichnung für die individuellen Gesundheitszustände auffassen lässt, die keinen darüber hinausgehenden normativen Gehalt besitzt: Der Einzelne ist nicht Bestandteil eines »Volkskörpers «, für dessen Gesundheitszustand er verantwortlich wäre.

Damit bleibt nur eine vierte Begründung, dass es um das gesundheitliche
Wohlergehen jedes einzelnen Bürgers gehe. Damit betritt die öffentliche Gewalt aber vermintes Gelände: Die Bürger in ihrem eigenen Interesse zu einer gesundheitsbewussteren Lebensführung zu drängen, zu erziehen oder gar zu zwingen, kollidiert mit dem Grundsatz, dass der freiheitliche Staat zur Neutralität gegenüber den unterschiedlichen

\section{Eine perfektionistische oder alistische Politik ist dem grundsätzlich versagt.}

Lebensformen und -entscheidungen verpflichtet ist (Huster 2002). Er darf regulierend eingreifen, soweit die Interessen Dritter oder der Allgemeinheit betroffen sind; eine darüber hinausgehende Bewertungskompetenz besitzt er aber nach verbreiteter Ansicht nicht. Eine perfektionistische oder paternalistische Politik ist ihm daher grundsätzlich versagt. Wie tief dieser Grundsatz auch in die verfassungsrechtliche Bewertung eingedrungen ist, wird dadurch belegt, dass sich das Bundesverfassungsgericht veranlasst gesehen hat, zur Rechtfertigung der gesetzlichen Rauchverbote in Gaststätten zu betonen: »Die Raucher werden hierbei nicht in unzulässiger Weise bevormundet, ihnen wird insbesondere kein Schutz vor Selbstgefährdung aufgedrängt. Die Landesnichtraucherschutzgesetze zielen weder auf Suchtprävention noch auf den Schutz des Einzelnen vor sich selbst. Ihr Ziel ist vielmehr der Schutz vor den Gefahren des Passivrauchens. Es geht um den Schutz der Gesundheit nicht des Rauchers, sondern der Gesundheit der anderen Personen, die in der jeweiligen Situation nicht selbst rauchen « (BVerfG 2008).

Dass diese Konstruktion des Bundesverfassungsgerichts zutreffend ist, kann man aus zwei Gründen bezweifeln. Zum einen ist nicht klar, wie der Nichtraucherschutz derartig weitgehende und flächendeckende Verbote soll rechtfertigen können, die das Gericht für zulässig hält (vgl. BVerfG 2008). Ginge es tatsächlich nur um den »Schutz der Gesundheit der anderen Personen, die in der jeweiligen Situation nicht selbst rauchen ", wäre es sehr viel naheliegender, ihnen aufzugeben, die Orte, an denen geraucht wird, zu meiden. Zum anderen fügen sich die 
Nichtraucherschutzgesetze in ein Ensemble von Maßnahmen ein, die das Rauchen als solches bekämpfen; es ist daher mehr als unwahrscheinlich, dass diesen Gesetzen jeder präventionspolitische Impetus fehlt. Das Verbot einer paternalistischen Politik scheint daher entweder der staatlichen Gesundheitspolitik die Hände zu binden oder zur Heuchelei zu zwingen.

\subsection{Die Public Health-Perspektive}

Für die Apologeten einer Public HealthPolitik ist dieses Ergebnis insbesondere mit Blick auf die sozialen Gesundheitsungleichheiten nicht zu akzeptieren (zur Diskussion vgl. nur Callahan 2000 und Wikler 2005). Zwar betonen sie, dass der soziale Gesundheitsgradient nur zum Teil den Lebensstilen in den verschiedenen sozialen Schichten zugeschrieben werden kann. Trotzdem verlöre die Politik ein Handlungsfeld mit erheblicher Bedeutung, müsste sie lebensführungsbezogene Gesundheitsfaktoren und -ungleichheiten ignorieren. An dieser Stelle maßgeblich auf das Prinzip der Eigenverantwortung zu setzen, liefe Gefahr, die Schuld den schon Benachteiligten zuzuweisen (»victim blaming «) und die öffentliche Gewalt aus ihrer Verantwortung dafür zu entlassen, die für gesundheitsbewusste Entscheidungen hilfreichen sozialen Strukturen zu fördern. Zudem zeigen die bisherigen Erfahrungen, dass Gewohnheiten der Lebensführung jedenfalls dann außerordentlich änderungsresistent sind, wenn sie in Form von Ermahnungen und Aufklärungskampagnen isoliert angegangen werden; der bloße Appell an die Selbstverantwortung der Bürger führt nicht sehr weit, wenn sich nicht auch die sozialen Rahmenbedingungen ändern.

Diese Aspekte weisen darauf hin und hier liegt der in normativer Hinsicht entscheidende Punkt -, dass individuelle Verhaltensweisen unterschiedlich stark von der kulturellen und sozialen Umwelt geprägt sein können. Lebensstile wie das Ernährungs- und Bewegungsverhalten werden häufig bereits in der Kindheit angelegt, durch soziale und mediale Einflüsse verstärkt und weisen nicht selten Suchtcharakter auf. Dies sind die typischen Elemente, die uns zur Zurückhaltung mahnen sollten, die Verantwortung für diese Verhal- tensweisen und ihre Folgen allein dem Individuum zuzuschreiben; es gibt hier offensichtlich »Ursachen der Ursachen «. Dass das jeweilige Verhalten nicht so frei ist, wie gelegentlich behauptet wird, erkennt man auch unschwer daran, dass viele Bürger ihre gesundheitsschädlichen Angewohnheiten ja selbst ändern wollen, es ihnen aber nicht gelingt. In dieser Situation kann eine Politik, die es ihnen erleichtert, ihren eigentlichen Willen handlungswirksam werden zu lassen (grundlegend dazu Frankfurt 1971), geradezu als Freiheitsförderung betrachtet werden (Bloche 2005).

\section{Individuelle Verhaltens-} weisen können unterschiedlich stark von der kulturellen und sozialen Umwelt geprägt sein.

Hier kommt eine grundsätzliche Spannung zwischen den Perspektiven des Public Health-Ansatzes als einer empirischen Sozialwissenschaft einerseits und des Rechts und der Sozialphilosophie andererseits zum Ausdruck. Während Recht und Gerechtigkeitstheorie von der individuellen Autonomie und ihrer Legitimationskraft ausgehen, ist einem Public Health-Ansatz diese Perspektive aus prinzipiellen Gründen fremd: Er schaut nicht auf das Individuum und die Freiheit seiner Willensbetätigung, sondern abstrahiert davon und stellt auf einer rein statistischen Ebene ein schichtenspezifisches Gesundheitsverhalten fest, obwohl die konkreten Mitglieder dieser Schicht wechseln. Dies legt den Gedanken nahe, dass nicht die Individuen selbst, sondern die sozialen Einflussfaktoren für diese Regelmäßigkeiten und die Unterschiede zwischen den sozialen Einheiten verantwortlich sind.

Nun muss diese Spannung zwischen einer Teilnehmerperspektive, in der wir uns selbst als freie und verantwortliche Akteure wahrnehmen und auch anderen diese Eigenschaft zuschreiben, und einer Beobachterperspektive, die den sozialen und kulturellen Hintergrund unserer Entscheidungen und unseres Verhaltens betont, nicht in einem unauflöslichen Gegensatz enden. Vielmehr sind wir es auch in anderen Lebensbereichen gewohnt, die individuellen Entschei- dungen zu respektieren, ohne deshalb behaupten zu müssen, dass Individuen unabhängig von diesen Hintergrundfaktoren existieren. Wie die Verantwortung zwischen Individuum und Gesellschaft aufgeteilt wird, muss dann allerdings das Gemeinwesen für jeden Sachbereich immer wieder neu aushandeln und festlegen (vgl. dazu Roemer 1993).

\subsection{Juristische Konsequenzen}

Vor diesem Hintergrund müssen Beobachter- und Teilnehmerperspektive auch im Recht zu einem Ausgleich gebracht werden. Dies dürfte zum einen bedeuten, dass das Recht die individuelle Entscheidungsfreiheit vor einem gesundheitspolitischen Aktivismus schützen muss. Aus der Anerkennung der Wirkungsmächtigkeit sozialer Faktoren folgt nicht, dass die individuellen Entscheidungen nicht respektiert werden müssten. Dass die sozialontologische These von der sozialen und kulturellen Bedingtheit individuellen Verhaltens richtig ist, gibt dem Gemeinwesen den Auftrag, diese Bedingungen zu pflegen; es gibt ihm aber in normativer Hinsicht nicht das Recht, dieses Verhalten zu unterbinden. Diese Differenzierung ist aus der sozialphilosophischen Diskussion zwischen liberalen und kommunitaristischen Theorien bekannt (vgl. dazu etwa Forst 1994). Angesichts des Umstandes, dass es hier um höchstpersönliche Aspekte der Lebensgestaltung geht, ist eine Präventions- und Gesundheitsförderungspolitik in einer freiheitlichen Ordnung weithin nur als Information, Beratung, Ermunterung und gesundheitsdienliche Ausgestaltung der Lebenswelten, typischerweise dagegen nicht in Form von rechtsverbindlichen Ge- und Verboten möglich: Wer sich bei hinreichender Information und Willensfreiheit für einen ungesunden Lebensstil entscheidet, muss sich in einem freiheitlichen $\mathrm{Ge}$ meinwesen nicht daran hindern lassen.

Zum anderen wird die Rechtsordnung aber auch anerkennen müssen, dass die Zuschreibung individueller Verantwortung nicht ohne Berücksichtigung der empirischen Zusammenhänge erfolgen muss und kann. Vermutlich wird man hier zu einer Abstufung der Art kommen, dass politische Interventionen umso eher möglich sind, je stärker der Verdacht ist, dass sich in einem ge- 
sundheitsriskanten Verhalten nicht das wohlverstandene Interesse des Individuums, sondern die sozialen Hintergrundfaktoren Bahn brechen.

\section{Der Zusammenhang von prospektiver und retrospektiver Verantwortung}

Der zuletzt erwähnte Punkt ist auch für das Verhältnis von Versorgungssystem und Public Health-Politik von Bedeutung. Die Zuschreibung einer retrospektiven Verantwortung für eine eingetretene Erkrankung - z. B. in Form der Kostenbeteiligung - ist umso problematischer, je weniger plausibel wir das entsprechende gesundheitsgefährdende Verhalten auf den Willen des Einzelnen gründen können und je stärker wir es auf soziale Faktoren zurückführen müssen. $\mathrm{Ob}$ und inwieweit dies der Fall ist, hängt insbesondere davon $\mathrm{ab}$, welche Anstrengungen das Gemeinwesen unternommen hat, die Möglichkeit der Übernahme der prospektiven Verantwortung für die eigene Gesundheit zu stärken (zur Begrifflichkeit vgl. Marckmann; Gallwitz 2007). Angesichts der bekannten empirischen Zusammenhänge ist es illegitim, Bürgern die Kosten ihrer Krankheit aufzuerlegen, die aufgrund von Bildungsdefiziten und ungünstigen Lebensumständen keine realistische Chance hatten, ein gesünderes Leben zu führen.

Ebenso gilt aber umgekehrt, dass die Vertreter einer scharfen Kritik staatlicher Präventionsbemühungen, die das Prinzip der individuellen Autonomie betonen, sich dann auch im Rahmen des Versorgungssystems konsequenterweise nicht dagegen sperren können, dass der Eigenverantwortung ein größeres Gewicht zugemessen wird. Dass dieser Weg die sozialen Unterschiede verstär ken dürfte, sollten gerade diejenigen bedenken, die einen vermeintlichen »healthism« der Public Health-Politik nicht aus liberalistischer, sondern aus bürgerrechtlicher Perspektive kritisieren.

\section{Literatur}

Alber K.; Kliemt H.; Nagel E. (2009), Selbstverantwortung als Kriterium kaum operationalisierbar. Deutsches Ärzteblatt 106: S A $1361-\mathrm{A} 1363$

Beske F. (2002), Prävention: Vor Illusionen wird gewarnt. Deutsches Ärzteblatt 99: S. A1209-A1210.

Bloche M.G. (2005), Obesity and the struggle within ourselves. The Georgetown LiveJourna 93: S. 1335-1359.

Bundesverfassungsgericht (2005) Beschluss v. 6.12.2005. 1 BVR 347/98

Bundesverfassungsgericht (2007) Beschluss v. 29.11.2007. 1 BvR 2496/07.

Bundesverfassungsgericht (2008) Urteil v. 30.7.2008. 1 BvR 3262/07, 402/08 und 906/08.

Burris S. (1997), The invisibility of public health. American Journal of Public Health 87 S. 1607-1610.

Buyx A. (2005), Eigenverantwortung als Verteilungskriterium im Gesundheitswesen. Ethik in der Medizin 17: S. 269-283.

Callahan D. (Hrsg) (2000), Promoting healthy behavior: how much freedom? Whose responsibility? Georgetown University Press, Washington DC

Daniels N. (1985), Just health care. Cambridge University Press, Cambridge.

Dworkin R. (1981), What is equality? Part 2: equality of resources. Philos Public Affairs 10 : S. $283-345$

Eichenhofer E. (2003), Wahl des Lebensstils - Auswirkungen in der sozialen Sicherheit. Sozialgerichtsbarkeit 50: S. 705-712.

Forst R. (1994), Kontexte der Gerechtigkeit. Suhrkamp, Frankfurt/M.

Frankfurt H.G. (1971), Freedom of the will and the concept of a person. Journal of Philosophy 68: S. 5-20.

Frenzel E.M. (2007), Die Volksgesundheit in der Grundrechtsdogmatik. Die Öffentliche Verwaltung: S. 243-248.
Gröschner R. (2008), Vom Ersatzgesetzgeber zum Ersatzerzieher. Zeitschrift für Gesetzgebung: S. 400-412.

Händeler E. (2008), Die echte Gesundheitsreform. Die BKK: S. 382-387.

Höfling W. (2009), Recht auf Selbstbestimmung versus Pflicht zur Gesundheit. Zeitschrift für Evidenz, Fortbildund und Qualität im Gesundheitswesen 103: S. 286-292.

Huster S. (2002), Die ethische Neutralität des Staates. Mohr/Siebeck, Tübingen

Huster S. (2008), Gesundheitsgerechtigkeit: Public Health im Sozialstaat. Juristenzeitung 63: S. 859-867.

Huster S.; Kliemt H. (2009), Opportunitätskosten und Jurisprudenz. Archiv für Rechtsund Sozialphilosophie 95: S. 241-251.

Marckmann G.; Gallwitz B. (2007), Gesundheitliche Eigenverantwortung beim Typ-2Diabetes. Zeitschrift für medizinische Ethik 53: S. 103-116

Mielck A. (2000), Soziale Ungleichheit und Gesundheit in Deutschland. Hans Huber, Bern

Muckel S. (2009), Sozialrecht, 3. Aufl. Beck, München.

Richter M.; Hurrelmann K. (Hrsg) (2006), Gesundheitliche Ungleichheit. Grundlagen, Probleme, Perspektiven. VS Verlag für Sozialwissenschaften, Wiesbaden.

Roemer J. (1993), A pragmatic theory of responsibility for the egalitarian planners. Philosophy and Public Affairs 22: S. 146-166.

Rose G. (1985), Sick individuals and sick populations. International Journal for Epidemiology 14: S. 32-38.

Wienke A. (2009), Eigenverantwortung der Patienten/Kunden: Wohin führt der Rechtsgedanke des $§ 52$ Abs. 2 SGB V?, in: Wienke A. Eberbach W.H., Kramer H.-J., Janke K. (Hrsg), Die Verbesserung des Menschen. Springer, Berlin, S. 169-177.

Wikler D. (2005), Personal and social responsibility for health, in: Anand S., Peter F., Sen A. (Hrsg), Public health, ethics, and equity. Oxford University Press, Oxford, S. 109-134. 